\title{
A NOTE ON EXACT LAWS OF LARGE NUMBERS FOR ASYMMETRIC PARETO-TYPE DISTRIBUTIONS WITH APPLICATIONS TO RATIOS OF RANDOM VARIABLES
}

\author{
PRZEMYSŁAW MATUŁA ${ }^{1, a}$, PAWEŁ KURASIŃSKI ${ }^{2, b}$, \\ HABIB NADERI ${ }^{3, c}$ AND ANDRÉ ADLER ${ }^{4, d}$ \\ ${ }^{1}$ Institute of Mathematics, Marie Curie-Skłodowska University, pl. M.C.-Skłodowskiej 1, 20-031 Lublin, \\ Poland. \\ ${ }^{a}$ E-mail: matula@hektor.umcs.lublin.pl \\ ${ }^{2}$ Institute of Mathematics, Marie Curie-Skłodowska University, pl. M.C.-Skłodowskiej 1, 20-031 Lublin, \\ Poland. \\ ${ }^{b}$ E-mail: pawelkurasinski1@gmail.com \\ ${ }^{3}$ Department of Mathematics, University of Sistan and Baluchestan, Zahedan, Iran. \\ ${ }^{c}$ E-mail: h.h.naderi@gmail.com \\ ${ }^{4}$ Department of Mathematics, Illinois Institute of Technology Chicago, Illinois, 60616, USA. \\ ${ }^{d}$ E-mail: adler@iit.edu
}

\|\|

\begin{abstract}
We prove some unusual limit laws for weighted sums of i.i.d. random variables with asymmetric Pareto-type distributions. The obtained results are applied to the convergence of ratios of independent random variables.
\end{abstract}

\section{Introduction}

Let us begin with the following example.

Example 1. Let us consider the asymmetric Laplace distribution with the following density function

$$
f(x)= \begin{cases}a \exp (-x), & x \geq 0 \\ b \exp (x), & x<0\end{cases}
$$

Received February 6, 2020 and in revised form June 15, 2020.

AMS Subject Classification: 60F15, 60F05, 60G50.

Key words and phrases: Law of large numbers, i.i.d. random variables, ratios of random variables. 
where $a, b \geq 0$ and $a+b=1$. In a special case $a=b=1 / 2$ we get the standard Laplace distribution with the density $f(x)=\frac{1}{2} \exp (-|x|)$. It is not hard to check that for independent and identically distributed random variables $X$ and $Y$ with the same asymmetric Laplace distribution with the density (1), their ratio $R=X / Y$ has the following distribution:

$$
F_{R}(r)= \begin{cases}1-\frac{a^{2}+b^{2}}{1+r}, & r \geq 0 \\ \frac{2 a b}{1-r}, & r<0 .\end{cases}
$$

Let us observe that the above distribution (2) satisfies the following limiting properties:

$$
\begin{aligned}
\lim _{x \rightarrow+\infty} x \bar{F}_{R}(x) & =\lim _{x \rightarrow+\infty} x \mathbb{P}(R>x)=p \\
\lim _{x \rightarrow-\infty}|x| F_{R}(x) & =\lim _{x \rightarrow+\infty} x \mathbb{P}(-R \geq x)=q,
\end{aligned}
$$

with $p=a^{2}+b^{2}$ and $q=2 a b$.

We shall say that the random variable $R$ satisfying (3) has the asymmetric Pareto-type distribution. It is easy to see that if (3) is satisfied then $\lim _{x \rightarrow \pm \infty} x \mathbb{P}(R=x)=0$.

The other examples of this kind are two tailed Pareto distribution with the density

$$
f(x)= \begin{cases}\frac{q}{x^{2}}, & x \leq-1 \\ 0, & -1<x<1 \\ \frac{p}{x^{2}}, & x \geq 1\end{cases}
$$

where $p, q \geq 0$ and $p+q=1$ or the asymmetrical Cauchy distribution with the density

$$
f(x)= \begin{cases}\frac{q}{\pi(1+x)^{2}}, & x<0 \\ \frac{p}{\pi(1+x)^{2}}, & x \geq 0\end{cases}
$$

where $p, q \geq 0$ and $p+q=2$.

Consider a sequence $\left(R_{n}\right)_{n \in \mathbb{N}}$ of i.i.d. random variables satisfying (3). These r.v.'s are not integrable and the classical laws of large numbers cannot be applied in this case. To obtain nontrivial limits for sums of such r.v.'s one must consider weighted sums. Limit theorems of this kind are called exact laws of large numbers and have been intensively studied in recent 
years (see [1] for the introduction to the problem and more recent paper [5] where dependent r.v.'s are considered). Strong and weak exact laws of large numbers for sequences satisfying (44) were studied in [4] and for sequences satisfying (5) in [3]. The most recent research in this direction may be found in [9] and [11]. Exact laws of large numbers found the applications to ratios of random variables and to ratios of order statistics (see [6], 7], 8] and [12] where further references are given).

The idea of exact laws grew from the fair games problem (see [6] pages 248-251). The idea is to balance the sum of random variables with constants, so that the limit in some sense is equal to one. The sum of these random variables can be considered the winning from some game, after $n$ plays of the game (see the St. Petersburg game, [6] pages 251-253). Meanwhile the norming sequence can be considered the amount the player should pay to play this game after $n$ trials. If the limit of the ratio of the winnings and the entrance fee converges to one it would make sense for both the house and the player to partake in this game.

In this paper we study the exact laws of large numbers for i.i.d. random variables with asymmetric Pareto-type distributions (i.e., satisfying (3)) which appear in a natural way for ratios of independent r.v.'s which are not necessarily positive. Let us mention that such problems have been studied in the literature only for ratios of positive r.v.'s (see [7] for further references).

As usual we use the notation $\lg x=\ln (\max (x, e))$, furthermore in the proofs $C>0$ is a generic constant which may change from one step to the other.

\section{Main Result}

Let us begin with the strong version of the exact law for random variables with asymmetric Pareto-type distributions. Note that in the symmetric case (if $p=q$ ) the result still holds, but it is not as interesting.

Theorem 1. Let $\left(R_{n}\right)_{n \in \mathbb{N}}$ be a sequence of i.i.d. random variables with the same distribution as a random variable $R$ satisfying (3). Then, for all $\beta>0$, we have

$$
\lim _{n \rightarrow \infty} \frac{1}{b_{n}} \sum_{k=1}^{n} a_{k} R_{k}=\frac{p-q}{\beta}, \text { almost surely }
$$


where $a_{n}=\left(\lg ^{\beta-2} n\right) / n$ and $b_{n}=\lg ^{\beta} n$.

Proof. Let $\varepsilon$ be given, from (3) it follows that there exists $x(\varepsilon)$ such that for $x>x(\varepsilon)$

$$
\begin{aligned}
& \frac{p-\varepsilon}{x} \leq \mathbb{P}(R>x) \leq \frac{p+\varepsilon}{x} \\
& \frac{q-\varepsilon}{x} \leq \mathbb{P}(R<-x) \leq \frac{q+\varepsilon}{x} .
\end{aligned}
$$

From (6]) and (7), for $x>x(\varepsilon)$ we get

$$
\frac{p+q-2 \varepsilon}{x} \leq \mathbb{P}(|R|>x) \leq \frac{p+q+2 \varepsilon}{x} .
$$

Note that, for $0<x \leq x(\varepsilon)$ we have

$$
x \mathbb{P}(|R|>x) \leq x(\varepsilon) .
$$

and therefore there exists a constant $M>0$ such that for $x>0$ we have

$$
\mathbb{P}(|R|>x) \leq \frac{x(\varepsilon)+2 \varepsilon+p+q}{x}=\frac{M}{x} .
$$

Let $c_{n}=b_{n} / a_{n}=n \lg ^{2} n$. We start with splitting our weighted sum into three terms

$$
\begin{aligned}
\frac{1}{b_{n}} \sum_{k=1}^{n} a_{k} R_{k}= & \frac{1}{b_{n}} \sum_{k=1}^{n} a_{k}\left[R_{k} \mathbb{I}\left(\left|R_{k}\right| \leq c_{k}\right)-\mathbb{E} R_{k} \mathbb{I}\left(\left|R_{k}\right| \leq c_{k}\right)\right] \\
& +\frac{1}{b_{n}} \sum_{k=1}^{n} a_{k} R_{k} \mathbb{I}\left(\left|R_{k}\right|>c_{k}\right)+\frac{1}{b_{n}} \sum_{k=1}^{n} a_{k} \mathbb{E} R_{k} \mathbb{I}\left(\left|R_{k}\right| \leq c_{k}\right) .
\end{aligned}
$$

By (9), we have

$$
\sum_{n=1}^{\infty} \frac{1}{c_{n}^{2}} \mathbb{E} R_{n}^{2} \mathbb{I}\left(\left|R_{n}\right| \leq c_{n}\right) \leq 2 \sum_{n=1}^{\infty} \frac{1}{c_{n}^{2}} \int_{0}^{c_{n}} t \mathbb{P}(|R|>t) d t \leq 2 M \sum_{n=1}^{\infty} \frac{1}{c_{n}}<\infty
$$

Therefore, we apply the two series theorem and Kronecker lemma to see that the first term in (10) converges almost surely to zero. Further, we check that 
$\sum_{n=1}^{\infty} \mathbb{P}\left(\left|R_{n}\right|>c_{n}\right)<\infty$, which follows from (9) and the estimate

$$
\sum_{n=1}^{\infty} \mathbb{P}\left(\left|R_{n}\right|>c_{n}\right) \leq \sum_{n=1}^{\infty} \frac{M}{c_{n}}<\infty .
$$

Therefore the almost sure convergence to zero of the second term in (10) follows from the Borel-Cantelli lemma. To end the proof it is sufficient to prove that (see proof of Theorem 1 in [1])

$$
\lim _{n \rightarrow \infty} \frac{1}{\lg n} \mathbb{E} R_{n} \mathbb{I}\left(\left|R_{n}\right| \leq c_{n}\right)=p-q .
$$

Let us denote by $R_{n}^{+}=\max \left(R_{n}, 0\right)$ and $R_{n}^{-}=\max \left(-R_{n}, 0\right)$ the positive and negative part of $R_{n}$. Let us observe that there exists $n_{0}$ such that for $n \geq n_{0}$ we have $c_{n} \geq x(\varepsilon)$. By (6) and (7), for $n \geq n_{0}$ we get

$$
\begin{aligned}
\frac{1}{\lg n} \mathbb{E} R_{n} \mathbb{I}\left(\left|R_{n}\right| \leq c_{n}\right)=\frac{1}{\lg n}\left(\mathbb{E} R_{n}^{+} \mathbb{I}\left(R_{n}^{+} \leq c_{n}\right)-\mathbb{E} R_{n}^{-} \mathbb{I}\left(R_{n}^{-} \leq c_{n}\right)\right) \\
=\frac{1}{\lg n}\left(-c_{n} \mathbb{P}\left(R_{n}>c_{n}\right)+\int_{0}^{c_{n}} \bar{F}_{R_{n}}(t) d t\right. \\
\left.\quad-\left(-c_{n} \mathbb{P}\left(R_{n}<-c_{n}\right)+\int_{0}^{c_{n}} F_{R_{n}}(-t) d t\right)\right) \\
=\frac{1}{\lg n}\left(\left(-c_{n} \mathbb{P}\left(R_{n}>c_{n}\right)+\int_{0}^{x(\varepsilon)} \bar{F}_{R_{n}}(t) d t+\int_{x(\varepsilon)}^{c_{n}} \bar{F}_{R_{n}}(t) d t\right)\right. \\
\left.\quad-\left(-c_{n} \mathbb{P}\left(R_{n}<-c_{n}\right)+\int_{0}^{x(\varepsilon)} F_{R_{n}}(-t) d t+\int_{x(\varepsilon)}^{c_{n}} F_{R_{n}}(-t) d t\right)\right) \\
\leq \frac{1}{\lg n}\left(-c_{n} \frac{p-\varepsilon}{c_{n}}+x(\varepsilon)+\left(\lg \left(c_{n}\right)-\lg x(\varepsilon)\right)(p+\varepsilon)\right. \\
\left.\quad+c_{n} \frac{q+\varepsilon}{c_{n}}-\left(\lg \left(c_{n}\right)-\lg x(\varepsilon)\right)(q-\varepsilon)\right) \\
\leq \frac{1}{\lg n}\left(-p+q+2 \varepsilon+x(\varepsilon)+(p-q+2 \varepsilon)\left(\lg c_{n}-\lg x(\varepsilon)\right)\right) .
\end{aligned}
$$

thus $\limsup _{n \rightarrow \infty} \frac{1}{\lg n} \mathbb{E} R_{n} \mathbb{I}\left(\left|R_{n}\right| \leq c_{n}\right) \leq(p-q+2 \varepsilon)$. Similarly we prove $\liminf _{n \rightarrow \infty} \frac{1}{\lg n} \mathbb{E} R_{n} \mathbb{I}\left(\left|R_{n}\right| \leq c_{n}\right) \geq(p-q-2 \varepsilon)$. Since $\varepsilon$ was arbitrary we get (11), in consequence $\frac{1}{b_{n}} \sum_{k=1}^{n} a_{k} \mathbb{E} R_{k} \mathbb{I}\left(\left|R_{k}\right| \leq c_{k}\right) \rightarrow \frac{p-q}{\beta}$ and the proof is completed. 
In the next theorem we will prove an analogue of the main result of [2] for asymmetric Pareto-type distributions.

Theorem 2. Let $\left(R_{n}\right)_{n \in \mathbb{N}}$ be a sequence of i.i.d. random variables with the same distribution as a random variable $R$ satisfying (3). Then for all $\varepsilon>0$ and $\beta>0$,

$$
\sum_{n=1}^{\infty} d_{n} \mathbb{P}\left(\left|\frac{1}{b_{n}} \sum_{k=1}^{n} a_{k} R_{k}-\frac{p-q}{\beta}\right|>\varepsilon\right)<\infty,
$$

where $a_{n}=\left(\lg ^{\beta-2} n\right) / n, b_{n}=\lg ^{\beta} n$ and $d_{n}=\frac{1}{n \lg n}$.

Proof. We split our weighted sum as in (10) $\frac{1}{b_{n}} \sum_{k=1}^{n} a_{k} R_{k}=: A_{1, n}+A_{2, n}+$ $A_{3, n}$. Let us observe that

$$
\begin{aligned}
& \sum_{n=1}^{\infty} d_{n} \mathbb{P}\left(\left|A_{1, n}+A_{2, n}+A_{3, n}-\frac{p-q}{\beta}\right|>\varepsilon\right) \\
& \leq \sum_{n=1}^{\infty} d_{n} \mathbb{P}\left(\left|A_{1, n}\right|>\frac{\varepsilon}{3}\right)+\sum_{n=1}^{\infty} d_{n} \mathbb{P}\left(\left|A_{2, n}\right|>\frac{\varepsilon}{3}\right) \\
& \quad+\sum_{n=1}^{\infty} d_{n} \mathbb{P}\left(\left|A_{3, n}-\frac{p-q}{\beta}\right|>\frac{\varepsilon}{3}\right) .
\end{aligned}
$$

As in the proof of Theorem 1 let us put $c_{n}=b_{n} / a_{n}=n \lg ^{2} n$.

Now we show that the term with $A_{1, n}$ is convergent. By Markov's inequality and (9)

$$
\begin{aligned}
& \sum_{n=1}^{\infty} d_{n} \mathbb{P}\left(\left|b_{n}^{-1} \sum_{k=1}^{n} a_{k}\left[R_{k} \mathbb{I}\left(\left|R_{k}\right| \leq c_{k}\right)-\mathbb{E} R_{k} \mathbb{I}\left(\left|R_{k}\right| \leq c_{k}\right)\right]\right|>\frac{\varepsilon}{3}\right) \\
& \leq C \sum_{n=1}^{\infty} \frac{d_{n}}{b_{n}^{2}} \sum_{k=1}^{n} a_{k}^{2} \mathbb{E} R_{k}^{2} \mathbb{I}\left(\left|R_{k}\right| \leq c_{k}\right) \leq 2 C \sum_{n=1}^{\infty} \frac{d_{n}}{b_{n}^{2}} \sum_{k=1}^{n} a_{k}^{2} \int_{0}^{c_{k}} t \mathbb{P}(|R|>t) d t \\
& \leq 2 C M \sum_{n=1}^{\infty} \frac{d_{n}}{b_{n}^{2}} \sum_{k=1}^{n} a_{k}^{2} c_{k}=2 C M \sum_{n=1}^{\infty} \frac{1}{(n \lg n) \lg ^{2 \beta} n} \sum_{k=1}^{n} \frac{\lg ^{2 \beta-2} k}{k}
\end{aligned}
$$

on account of (see [2])

$$
\frac{1}{\lg ^{2 \beta} n} \sum_{k=1}^{n} \frac{\lg ^{2 \beta-2} k}{k} \leq \begin{cases}C / \lg ^{2 \beta} n, & \text { if } 0<\beta<1 / 2 \\ C \lg (\lg n) / \lg n, & \text { if } \beta=1 / 2 \\ C / \lg n, & \text { if } \beta>1 / 2\end{cases}
$$


we get $\sum_{n=1}^{\infty} d_{n} \mathbb{P}\left(\left|A_{1, n}\right|>\varepsilon / 3\right)<\infty$. To prove the convergence of the third term, we see that, as in the proof of Theorem $1, A_{3, n}$ is a nonrandom sequence which converges to $\frac{p-q}{\beta}$, therefore only finite number of $\mathbb{P}\left(\left|A_{3, n}-\frac{p-q}{\beta}\right|>\frac{\varepsilon}{3}\right)$ are nonzero. To prove the convergence of the second term we use Theorem 1 in [10] with

$$
X_{n k}=a_{k} R_{k} \mathbb{I}\left(\left|R_{k}\right|>c_{k}\right) / b_{n}
$$

Now we show that $\sum_{n=1}^{\infty} d_{n} \sum_{k=1}^{n} \mathbb{P}\left(\left|X_{n k}\right|>\varepsilon\right)<\infty$.

$$
\begin{aligned}
& \sum_{n=1}^{\infty} d_{n} \sum_{k=1}^{n} \mathbb{P}\left(\left|X_{n k}\right|>\varepsilon\right)=\sum_{n=1}^{\infty} d_{n} \sum_{k=1}^{n} \mathbb{P}\left(\left|R_{k}\right|>\max \left(c_{k}, \frac{\varepsilon b_{n}}{a_{k}}\right)\right) \\
& \left.\leq \sum_{n=1}^{\infty} d_{n} \sum_{k=1}^{n} \mathbb{P}\left(\left|R_{k}\right|>\frac{\varepsilon b_{n}}{a_{k}}\right)=\sum_{n=1}^{\infty} d_{n} \sum_{k=1}^{n} \mathbb{P}\left(\left|R_{k}\right|>\varepsilon k \lg ^{2-\beta} k \lg ^{\beta} n\right)\right) .
\end{aligned}
$$

by (9) and (12)

$$
\leq \sum_{n=1}^{\infty} d_{n} \sum_{k=1}^{n} \frac{M}{\varepsilon k \lg ^{2-\beta} k \lg ^{\beta} n}<\infty
$$

Next we show that $\sum_{n=1}^{\infty} d_{n}\left(\sum_{k=1}^{n} \mathbb{E} X_{n k}^{2} \mathbb{I}\left[\left|X_{n k}\right|<\delta\right]\right)^{J}<\infty$ with $J=2$ and $\delta=1$. By (9) and (12), we have

$$
\begin{aligned}
\sum_{n=1}^{\infty} & d_{n}\left(\sum_{k=1}^{n} \mathbb{E} X_{n k}^{2} \mathbb{I}\left[\left|X_{n k}\right|<1\right]\right)^{2} \\
& =\sum_{n=1}^{\infty} d_{n}\left(\sum_{k=1}^{n} \frac{a_{k}^{2}}{b_{n}^{2}} \mathbb{E} R_{k}^{2} \mathbb{I}\left[c_{k}<\left|R_{k}\right|<\frac{b_{n}}{a_{k}}\right]\right)^{2} \\
& \leq \sum_{n=1}^{\infty} d_{n}\left(\sum_{k=1}^{n} \frac{a_{k}^{2}}{b_{n}^{2}} \mathbb{E} R_{k}^{2} \mathbb{I}\left[\left|R_{k}\right|<\frac{b_{n}}{a_{k}}\right]\right)^{2} \\
\leq & C \sum_{n=1}^{\infty} d_{n}\left(\sum_{k=1}^{n} \frac{a_{k}^{2}}{b_{n}^{2}} \int_{0}^{\frac{b_{n}}{a_{k}}} t \mathbb{P}(|R|>t) d t\right)^{2} \\
\leq & C \sum_{n=1}^{\infty} d_{n}\left(\sum_{k=1}^{n} \frac{a_{k}^{2}}{b_{n}^{2}} \frac{b_{n}}{a_{k}}\right)^{2}=C \sum_{n=1}^{\infty} d_{n}\left(\sum_{k=1}^{n} \frac{\lg ^{\beta-2} k}{k \lg ^{\beta} n}\right)^{2} \\
& =C \sum_{n=1}^{\infty} \frac{1}{n \lg ^{2 \beta+1} n}\left(\sum_{k=1}^{n} \frac{\lg ^{\beta-2} k}{k}\right)^{2}<\infty
\end{aligned}
$$


Finally, we show that $\sum_{k=1}^{n} \mathbb{E} X_{n k} \mathbb{I}\left[\left|X_{n k}\right| \leq \delta \mid\right] \rightarrow 0$ as $n \rightarrow \infty$ with $\delta=1$.

Again, due to (9) and (12) we get

$$
\begin{aligned}
& \sum_{k=1}^{n} \mathbb{E} X_{n k} \mathbb{I}\left[\left|X_{n k}\right| \leq 1\right] \leq \sum_{k=1}^{n} \frac{a_{k}}{b_{n}} \mathbb{E}\left|R_{k}\right| \mathbb{I}\left[c_{k}<\left|R_{k}\right| \leq \frac{b_{n}}{a_{k}}\right] \\
& \quad \leq \sum_{k=1}^{n} \frac{a_{k}}{b_{n}} \mathbb{E}\left|R_{k}\right| \mathbb{I}\left[\left|R_{k}\right| \leq \frac{b_{n}}{a_{k}}\right] \leq \sum_{k=1}^{n} \frac{a_{k}}{b_{n}} \int_{0}^{\frac{b_{n}}{a_{k}}} \mathbb{P}(|R|>t) d t \\
& \quad=\sum_{k=1}^{n} \frac{a_{k}}{b_{n}}\left(\int_{0}^{1} \mathbb{P}(|R|>t) d t+\int_{1}^{\frac{b_{n}}{a_{k}}} \mathbb{P}(|R|>t) d t\right) \\
& \leq \sum_{k=1}^{n} \frac{a_{k}}{b_{n}}\left(1+M \lg \frac{b_{n}}{a_{k}}\right) \leq C \sum_{k=1}^{n} \frac{a_{k}}{b_{n}} \lg b_{n} \\
& \quad=\frac{C \lg (\lg n)}{\lg ^{\beta} n} \sum_{k=1}^{n} \frac{\lg \beta^{\beta-2} k}{k} \rightarrow 0, \text { as } n \rightarrow \infty .
\end{aligned}
$$

and the proof is completed.

For the sake of completeness let us state last theorem concerning convergence in probability in our exact laws. This result is due to Nakata (see Corollary 4.1 in [9]).

Theorem 3. Let $\left(R_{n}\right)_{n \in \mathbb{N}}$ be a sequence of i.i.d. random variables with the same distribution as a random variable $R$ satisfying (3), then for all $\beta>-1$ and any slowly varying function $L$, we have

$$
\lim _{n \rightarrow \infty} \frac{1}{b_{n}} \sum_{k=1}^{n} a_{k} R_{k}=\frac{p-q}{\beta+1}, \text { in probability, }
$$

where $a_{n}=n^{\beta} L(n), b_{n}=n^{\beta+1} L(n) \lg n$.

\section{Applications}

In this section we present the applications of the results from Section 2 to ratios of independent random variables which are not necessarily positive. We shall extend Example 1 from Section 1. Let $X$ and $Y$ be independent random variables with the same distribution as a random variable $\xi$ with 
bounded density $f(x)$. Suppose that the following limits exist

$$
\lim _{x \rightarrow 0^{+}} f(x)=f\left(0^{+}\right) \text {and } \lim _{x \rightarrow 0^{-}} f(x)=f\left(0^{-}\right)
$$

furthermore let $\xi$ be integrable and $\xi^{+}$and $\xi^{-}$denote its positive and negative part. Let us observe that in this case (3) is satisfied. To see this let $r>0$, then

$$
\begin{aligned}
F_{R}(r) & =\mathbb{P}\left(\frac{X}{Y} \leq r\right)=\iint_{\frac{x}{y} \leq r} f(x) f(y) d x d y \\
& =1-\int_{0}^{\infty} \int_{0}^{\frac{x}{r}} f(x) f(y) d x d y-\int_{-\infty}^{0} \int_{\frac{x}{r}}^{0} f(x) f(y) d x d y \\
& =1-\frac{1}{r} \int_{0}^{\infty} f(x)\left(\int_{0}^{x} f\left(\frac{t}{r}\right) d t\right) d x-\frac{1}{r} \int_{-\infty}^{0} f(x)\left(\int_{x}^{0} f\left(\frac{t}{r}\right) d t\right) d x
\end{aligned}
$$

thus under our assumptions

$$
\lim _{r \rightarrow+\infty} r \bar{F}_{R}(r)=\mathbb{E} \xi^{+} f\left(0^{+}\right)-\mathbb{E} \xi^{-} f\left(0^{-}\right) .
$$

Similarly, for $r<0$, we have

$$
\begin{aligned}
F_{R}(r) & =\mathbb{P}\left(\frac{X}{Y} \leq r\right)=\iint_{\frac{x}{y} \leq r} f(x) f(y) d x d y \\
& =\int_{0}^{\infty} \int_{\frac{x}{r}}^{0} f(x) f(y) d x d y+\int_{-\infty}^{0} \int_{0}^{\frac{x}{r}} f(x) f(y) d x d y \\
& =-\frac{1}{r} \int_{0}^{\infty} f(x)\left(\int_{0}^{x} f\left(\frac{t}{r}\right) d t\right) d x-\frac{1}{r} \int_{-\infty}^{0} f(x)\left(\int_{x}^{0} f\left(\frac{t}{r}\right) d t\right) d x
\end{aligned}
$$

and

$$
\lim _{r \rightarrow-\infty} r F_{R}(r)=-\mathbb{E} \xi^{+} f\left(0^{-}\right)+\mathbb{E} \xi^{-} f\left(0^{+}\right)
$$

For ratios described above we can apply the results of the previous section what is stated in the following theorem.

Theorem 4. Let $\left(X_{n}\right)_{n \in \mathbb{N}}$ and $\left(Y_{n}\right)_{n \in \mathbb{N}}$ be two sequences of i.i.d. random variables with the same distribution as some random variable $\xi$. Assume that these sequences are independent of each other. Suppose that $\xi$ has bounded density satisfying (13) and assume that $\mathbb{E}|\xi|<\infty$. Let $R_{n}=X_{n} / Y_{n}$ then for 
the sequence $\left(R_{n}\right)_{n \in \mathbb{N}}$ the conclusions of Theorem 1,2 and 3 are satisfied.

\section{References}

1. A. Adler, Exact strong laws, Bull. Inst. Math. Acad. Sinica, 28 (2000), 141-166.

2. A. Adler, Complete exact laws, Probab. Math. Statist. 20 (2000), 215-222.

3. A. Adler, Laws of large numbers for asymmetrical Cauchy random variables, J. Appl. Math. Stoch. Anal. (2007), Art. ID 56924, 6 pp.

4. A. Adler, Laws of large numbers for two tailed Pareto random variables, Probab. Math. Statist., 28 (2008), 121-128.

5. A. Adler, P. Matuła, On exact strong laws of large numbers under general dependence conditions, Probab. Math. Statist. 38 (2018), 103-121.

6. W. Feller, An Introduction to Probability Theory and Its Applications, Volume 1, Third Edition, John Wiley and Sons, 1968.

7. P. Matuła, A. Adler, P. Kurasiński, On exact strong laws of large numbers for ratios of random variables and their applications, Comm. Statist. Theory Methods, (2019) DOI: $10.1080 / 03610926.2019 .1586935$.

8. P. Matuła, P. Kurasiński and A. Adler, Exact strong laws of large numbers for ratios of the smallest order statistics, Statist. Probab. Lett., 152 (2019), 69-73.

9. T. Nakata, Weak laws of large numbers for weighted independent random variables with infinite mean, Statist. Probab. Lett., 109 (2016), 124-129.

10. S. H. Sung, A. I. Volodin, T. C. Hu, More on complete convergence for arrays, Statist. Probab. Lett., 71 (2005), 303-311.

11. H. Xu, X. Li, W. Yang, F. Xu, Laws of large numbers with infinite mean, J. Math. Inequal., 13 (2019), 335-349.

12. S. Xu, C. Mei, Y. Miao, Limit theorems for ratios of order statistics from uniform distributions, J. Inequal. Appl., (2019), DOI: 10.1186/s13660-019-2256-7. 